\title{
Re: Renal Artery Stenting is no Longer Indicated After ASTRAL: Pros and Cons
}

\author{
Jim A. Reekers
}

Published online: 8 February 2011

(c) The Author(s) 2011. This article is published with open access at Springerlink.com

The lack of randomized, controlled trials (RCT) for interventional radiology (IR) is one of the major problems of IR, which has been discussed for many years (SIR Dotterlecture 2008). The reasons for this are simple but difficult to overcome. IR does not control the patients, so they often do not control the trials. Second, performing a RCT is expensive and funding for device trials is very difficult, because there is still no regulatory need to produce RCT data for registration of a device. Finally, the IR market is small compared with, for example, interventional cardiology, and the industry is not willing to invest large sums, which are needed for a RCT. That is why we always see these small, underpowered, one-arm studies in IR, never reaching any level of evidence. The ASTRAL investigators were able to overcome all of these hurdles and should absolutely be congratulated for this. However, the question remains how they were able to do this. I think personal drive and commitment can be recognized, but there is another method that they used, which is now the basis of the ongoing discussion around the trial. Next to personal commitment, the most often used method to recruit patients is to "soften" the inclusion criteria, to make them more acceptable, under the umbrella of otherwise being unethical. For this the authors introduced the so-called "uncertainty principle" $[1,2]$. They state that it is widely used and even have a reference for this. It has never been used as far as I know; I only know of the Heisenberg uncertainty

\footnotetext{
J. A. Reekers $(\square)$

Department of Radiology, Academic Medical Center,

Amsterdam, The Netherlands

e-mail: j.a.reekers@amc.uva.nl
}

principle, which plays a role in quantum mechanics, to state the precise inequalities of certain pairs of physical properties, but this is probably something else. In their reference, a Lancet publication from 1998, the word uncertainly is mentioned three times in completely different contexts, and the uncertainty principle is not anywhere in this paper. The core of the discussion is that in the ASTRAL trial patients could be included when the doctor was uncertain whether the patient needed to be treated with a stent. The problem is that there are currently no scientific data for any doctor to be certain about this, which was just the motivation for doing the ASTRAL trial. Therefore, patient selection in the trial is based on the doctor's gut feeling, or any other personal reason. The authors further state that this so-called uncertainty principle resulted in a heterogeneous patient population. To my opinion, it only resulted in serious patient selection bias. This uncertainty could easily have been taken away if those patients who received a stent, because the doctor was certain that they needed one, were followed clinically to be certain that the right decision was made. This was, however, not done, which is a major flaw. The authors state that many are confused about the methodology and results of the ASTRAL trial. I think there is no confusion at all; the methodology and results are crystal clear. The conclusion of the ASTRAL trial should be that if you, as a wise doctor, think or feel that a patient needs to be treated with a stent, you should do so, but if you are uncertain, which seems to be rare looking at the ASTRAL inclusion data, the patient should get best medical treatment. I am sure that the ASTRAL investigators agree with this conclusion; however, nephrologists and other referring specialists read it completely different because for them it says that there is no advantage from renal stenting above best medical treatment. That these words are coming directly from the 
horse his mouth is not very helpful, as we can see in our everyday practise.

\section{Conflict of interest None.}

Open Access This article is distributed under the terms of the Creative Commons Attribution Noncommercial License which permits any noncommercial use, distribution, and reproduction in any medium, provided the original author(s) and source are credited.

\section{Reference}

1. The ASTRAL Trial Investigators (2009) Revascularization versus best medical therapy for renal artery stenosis. N Engl J Med 361:1952-1962

2. Chalmers N, Moss J, Nicholson AA (2011) Re: Renal artery stenting is no longer indicated after ASTRAL: pros and cons. Cardiovasc Intervent Radiol. doi:10.1007/s00270-011-0113-2 